\title{
Gamma Knife surgery for intracranial chordoma and chondrosarcoma: radiosurgical perspectives and treatment outcomes
}

\author{
Clinical article
}

\author{
Ji Hee Kim, M.D., ${ }^{1}$ Hyun Ho Jung, M.D., ${ }^{2}$ Jong Hee Chang, M.D., Ph.D., 2 \\ Jin Woo Chang, M.D., Ph.D., ${ }^{2}$ Yong Gou Park, M.D., Ph.D., ${ }^{2}$ \\ and Won Seok Chang, M.D. ${ }^{2}$
}

${ }^{1}$ Department of Neurosurgery, Hallym University Sacred Heart Hospital, Anyang, Gyeonggi-do; and ${ }^{2}$ Division of Stereotactic and Functional Neurosurgery, Yonsei Gamma Knife Center, Department of Neurosurgery, Yonsei University College of Medicine, Seoul, Republic of Korea

\begin{abstract}
Object. Intracranial chordomas and chondrosarcomas are histologically low-grade, locally invasive tumors that are reported to be similar in terms of anatomical location, clinical presentation, and radiological findings but different in terms of behavior and outcomes. The purpose of this study was to investigate and compare clinical outcomes after Gamma Knife surgery (GKS) for the treatment of intracranial chordoma and chondrosarcoma.

Methods. The authors conducted a retrospective review of the results of radiosurgical treatment of intracranial chordomas and chondrosarcomas. They enrolled patients who had undergone GKS for intracranial chordoma or chondrosarcoma at the Yonsei Gamma Knife Center, Yonsei University College of Medicine, from October 2000 through June 2007. Analyses included only patients for whom the disease was pathologically diagnosed before GKS and for whom more than 5 years of follow-up data after GKS were available. Rates of progression-free survival and overall survival were analyzed and compared according to tumor pathology. Moreover, the association between tumor control and the margin radiation dose to the tumor was analyzed, and the rate of tumor volume change after GKS was quantified.

Results. A total of 10 patients were enrolled in this study. Of these, 5 patients underwent a total of 8 sessions of GKS for chordoma, and the other 5 patients underwent a total of 7 sessions of GKS for chondrosarcoma. The 2- and 5 -year progression-free survival rates for patients in the chordoma group were $70 \%$ and $35 \%$, respectively, and rates for patients in the chondrosarcoma group were $100 \%$ and $80 \%$, respectively (log-rank test, $\mathrm{p}=0.04$ ). The 2 - and 5 -year overall survival rates after GKS for patients in the chordoma group were $87.5 \%$ and $72.9 \%$, respectively, and rates for patients in the chondrosarcoma group were $100 \%$ and $100 \%$, respectively (log-rank test, $\mathrm{p}=0.03)$. The mean rates of tumor volume change 2 years after radiosurgery were $79.64 \%$ and $39.91 \%$ for chordoma and chondrosarcoma, respectively $(p=0.05)$. No tumor progression was observed when margin doses greater than $16 \mathrm{~Gy}$ for chordoma and 14 Gy for chondrosarcoma were prescribed.

Conclusions. Outcomes after GKS were more favorable for patients with chondrosarcoma than for those with chordoma. The data also indicated that at 2 years after GKS, the rate of volume change is significantly higher for chordomas than for chondrosarcomas. The authors conclude that radiosurgery with a margin dose of more than 16 Gy for chordomas and more than 14 Gy for chondrosarcomas seems to enhance local tumor control with relatively few complications. Further studies are needed to determine the optimal dose of GKS for patients with intracranial chordoma or chondrosarcoma.
\end{abstract}

(http://thejns.org/doi/abs/10.3171/2014.7.GKS141213)

\section{KEY WORDS • chondrosarcoma • chordoma • Gamma Knife surgery • recurrence • survival • stereotactic radiosurgery}

$\mathrm{C}$ HORDOMAS and chondrosarcomas are very rare, slow-growing, and highly infiltrating tumors that commonly arise at the base of the skull. Chordomas arise from remnants of the primitive notochord and

Abbreviation used in this paper: GKS = Gamma Knife surgery. typically involve the cranial base, vertebrae, or sacrococcygeal regions, $, 2,17,25,30$ whereas chondrosarcomas are radiologically similar tumors that originate from primitive mesenchymal cells or the embryonic rest of the cartilaginous matrix of the cranium in the craniocervical regions or skull base..$^{2,17,25,30}$ Because of similarities in their clinical presentations, radiological features, and anatomical 


\section{Radiosurgery for intracranial chordoma and chondrosarcoma}

locations, chordomas and chondrosarcomas can be difficult to distinguish without pathological examination. , $25,30^{2}$ However, because their clinical behaviors, responses to treatment, and outcomes differ quite substantially, ${ }^{2,4,17}$ it is therefore likely that the optimal treatment approaches for these tumors also differ.

Although resection is currently the gold standard treatment for both of these tumors, Gamma Knife surgery (GKS) has also been reported to play a role as an adjuvant treatment modality. GKS is used to treat remnant or recurrent chordoma and chondrosarcoma, and a number of studies have demonstrated acceptable tumor control with low complication rates. $4,7,12,15,16,19,20,22$ However, despite a strong possibility that treatment outcomes will differ, and considering the differences in biological behavior of these malignancies, little is currently known about the differences in the results of radiosurgical treatment.

In this study, we retrospectively investigated the clinical and radiosurgical results for patients who had undergone GKS for intracranial chordoma and chondrosarcoma, and we compared the outcomes among these patients. Being able to predict GKS-induced changes in tumor volume over time may help differentiate between intracranial chordoma and chondrosarcoma if differentiation is not possible by using other methods (MRI).

\section{Methods}

Data for patients who consecutively underwent GKS for intracranial chordoma or chondrosarcoma at our institution (Yonsei Gamma Knife Center, Yonsei University College of Medicine) from October 2000 through June 2007 were retrospectively reviewed. Patients were included in the study if they had received a pathologically confirmed diagnosis of chordoma or chondrosarcoma based on previous resection or biopsy and if more than 5 years of post-GKS follow-up data were available. Patients were excluded from the study if they had undergone conventional fractionated radiotherapy before or just after GKS. GKS was performed by using a model B, C, or Perfexion Leksell Gamma Knife (Elekta AB). For each group of patients, clinical and radiological data and treatment outcomes were investigated. For all patients, follow-up clinical and neurological examinations were performed 1 month after GKS and every 6 or 12 months thereafter. A follow-up imaging study was usually conducted 6 and 12 months after GKS and annually thereafter. For patients with clinical symptoms or radiological evidence of progression, more frequent follow-up was conducted. Tumor volume measurements were performed by using axial T1-weighted Gd-enhanced MRI, and Leksell GammaPlan software version 10.0.0 (Elekta AB). The tumor response to GKS was assessed according to the Macdonald criteria ${ }^{29}$ as follows: complete response was defined as complete disappearance of the lesion, partial response was defined as more than $50 \%$ decrease in tumor volume on follow-up images, progressive disease was defined as more than a $25 \%$ increase in tumor volume or the appearance of new lesions on follow-up images, and stable disease was defined as less than $50 \%$ reduction in volume or less than $25 \%$ increase in volume.
The rates of progression-free survival and overall survival for chordoma and chondrosarcoma patients were calculated by using the Kaplan-Meier method and compared by using log-rank statistics. The rate of tumor volume change was calculated at 6 months and 2 years after GKS. The Mann-Whitney U-test was used to determine whether the mean rates of volume change differed between patients in the chordoma and chondrosarcoma groups. All statistical analyses were performed by using standard statistical processing software (SPSS version 20.0; SPSS Inc.). A probability value of less than 0.05 was defined as statistically significant.

This study was approved by the institutional review board of the local hospital and conducted in accordance with the Declaration of Helsinki. Informed consent was obtained from all patients.

\section{Results}

Patient demographics, radiosurgical treatment parameters, and tumor outcomes are summarized in Tables 1,2 , and 3 . A total of 10 patients who underwent 15 sessions of GKS were included in this study: 5 patients $(8$ sessions) with chordoma and 5 patients (7 sessions) with chondrosarcoma (Table 1). For patients in the chordoma and chondrosarcoma groups, mean ages were 49.40 and 51.00 years, respectively, and mean follow-up periods were 53.29 and 83.71 months, respectively.

Three patients in the chordoma group and all patients in the chondrosarcoma group had undergone 1 or more tumor resections by craniotomy before GKS. Two patients in the chordoma group had undergone tumor resection by the transsphenoidal approach. No patient had previously received chemotherapy. The most common presenting symptom was persistent headache (3 patients). One patient had short stature because of suprasellar chordoma. The mean target volume at GKS was $10.67 \mathrm{~cm}^{3}$ for chordoma and $14.54 \mathrm{~cm}^{3}$ for chondrosarcoma. The mean prescription dose applied to the tumor margin was 20.06 Gy for chordoma and 14.50 Gy for chondrosarcoma, and the mean maximal dose was 40.13 Gy for chordoma and 29.00 Gy for chondrosarcoma (Table 3). No significant differences in patient demographics and radiosurgical treatment parameters were found between the 2 groups.

\section{Tumor Control}

According to the latest follow-up information available, among patients with chordoma, 2 (40\%) were alive and $3(60 \%)$ had died (1 because of treated tumor progression, 1 because of tumor progression and multiple tumor metastases, and 1 because of sepsis resulting from compression of the brainstem by the tumor). Among patients with chondrosarcoma, $3(60 \%)$ were alive and $2(40 \%)$ had died ( 1 because of treated tumor progression and the other because of unrelated disease without evidence of tumor progression). The 2- and 5-year progression-free survival rates were $70 \%$ and $35 \%$, respectively, for the chordoma patients and $100 \%$ and $80 \%$, respectively, for the chondrosarcoma patients $(\mathrm{p}=0.04$, log-rank test, Fig. 1). The 2- and 5-year overall survival rates after GKS 
TABLE 1: Characteristics of patients, tumors, and radiosurgical treatment parameters*

\begin{tabular}{|c|c|c|c|c|c|c|c|c|}
\hline Case No. & Session No. & Age (yrs), Sex & Pathology & $\begin{array}{l}\text { Pre-GKS } \\
\text { Treatment }\end{array}$ & $\begin{array}{l}\text { Tumor Vol } \\
\left(\mathrm{cm}^{3}\right)\end{array}$ & $\begin{array}{c}\text { Prescribed } \\
\text { Margin Dose (Gy) }\end{array}$ & $\begin{array}{c}\text { Maximal Dose } \\
\text { (Gy) }\end{array}$ & No. of Isocenters \\
\hline 1 & 1 & $70, \mathrm{M}$ & $C D$ & $\mathrm{CO}$ & 5 & 13 & 26 & 24 \\
\hline 2 & 3 & $15, \mathrm{~F}$ & $C D$ & $\mathrm{CO}$ & 20.2 & 14.5 & 29 & 16 \\
\hline 3 & 4 & $75, \mathrm{M}$ & $C D$ & TSA & 18 & 35 & 70 & 8 \\
\hline 4 & 5 & $42, \mathrm{M}$ & $C D$ & $\mathrm{CO}$ & 22.2 & 17.5 & 35 & 31 \\
\hline \multirow[t]{2}{*}{5} & 7 & $45, F$ & $C D$ & TSA & 6.5 & 25 & 50 & 25 \\
\hline & 8 & & $C D$ & none & 0.6 & 22.5 & 45 & 1 \\
\hline 6 & 9 & $48, F$ & CS & $\mathrm{CO}$ & 5.8 & 15.3 & 30.5 & 8 \\
\hline 7 & 10 & $60, \mathrm{M}$ & CS & $\mathrm{CO}$ & 2.7 & 14 & 28 & 1 \\
\hline 8 & 11 & $32, \mathrm{M}$ & CS & $\mathrm{CO}$ & 23.4 & 14.3 & 28.5 & 27 \\
\hline 10 & 15 & $71, \mathrm{~F}$ & CS & $\mathrm{CO}$ & 21.2 & 15 & 30 & 27 \\
\hline
\end{tabular}

* $\mathrm{CD}=$ chordoma; $\mathrm{CO}=$ craniotomy; $\mathrm{CS}=$ chondrosarcoma; $\mathrm{TSA}=$ transsphenoidal approach.

were $87.5 \%$ and $72.9 \%$, respectively, for the chordoma patients and $100 \%$ and $100 \%$, respectively, for the chondrosarcoma patients $(\mathrm{p}=0.03$, log-rank test, Fig. 2).

Analysis of the 5 tumors that progressed (4 lesions in chordoma patients and 1 lesion in a chondrosarcoma patient) revealed that all 5 tumors progressed adjacent to the prescribed tumor volume; there were no distant tumor progressions or metastases. Among these 4 chordoma patients, tumor progression was noted in patients who received $14,14,15$, and $15 \mathrm{~Gy}$ as the dose to the margin of the tumor, whereas in the patient with progressive chondrosarcoma, the dose was 13 Gy (Fig. 3). Ac- cordingly, there was no tumor progression in patients in the chondrosarcoma and chordoma groups when margin doses were higher than 14 and 16 Gy, respectively.

The mean rate of volume change after 6 months was $57.50 \%$ for chordoma patients and $33.05 \%$ for chondrosarcoma patients $(\mathrm{p}=0.46)$, and the corresponding 2 -year rates were $79.64 \%$ and $39.91 \%$, respectively $(p=0.05$; Fig. 4).

\section{Complications}

Radiation-related complications occurred in 1 (10\%) patient, who experienced a transient complication after

TABLE 2: Outcomes after radiosurgical treatment ${ }^{*}$

\begin{tabular}{cclclc}
\hline Case No. & Session No. & Tumor Outcome & $\begin{array}{c}\text { Time to Progression } \\
(\mathrm{mos})\end{array}$ & $\begin{array}{c}\text { Radiation-Associated } \\
\text { Complications }\end{array}$ & $\begin{array}{c}\text { Follow-Up Duration } \\
(\text { mos })\end{array}$ \\
\hline 1 & 1 & progression & 20 & none & 65 \\
& 2 & progression & 36 & none & 44 \\
3 & 3 & partial response & NA & none & 97 \\
4 & 4 & partial response & NA & diplopia, gait disturbance & 109 \\
& 5 & partial response & NA & none & 8 \\
5 & 6 & progression & 5 & none & 7 \\
& 7 & progression & 30 & none & 43 \\
6 & 8 & stable & NA & none & 28 \\
7 & 9 & partial response & NA & none & 163 \\
9 & 10 & partial response & NA & none & 130 \\
9 & 11 & stable & NA & none & 101 \\
& 12 & partial response & NA & none & 68 \\
& 13 & progression & 58 & none & 58 \\
& 14 & stable & NA & none & 47 \\
\hline
\end{tabular}

* NA = not applicable. 
Radiosurgery for intracranial chordoma and chondrosarcoma

TABLE 3: Summary of patient characteristics and radiosurgical treatment parameters

\begin{tabular}{lccc}
\hline \multicolumn{1}{c}{ Characteristic } & Chordoma* $^{*}$ & Chondrosarcoma* & p Value \\
\hline no. of patients & 5 & 5 \\
no. of sessions & 8 & 7 & \\
M/F ratio & $3: 2$ & $2: 3$ & 1.000 \\
patient age at GKS (yrs) & $49.40(27.00-68.00)$ & $51.00(40.00-61.99)$ & 0.152 \\
follow-up duration (mos) & $53.29(26.15-82.00)$ & $83.71(47.58-120.84)$ & 0.232 \\
margin dose (Gy) & $20.06(15.50-25.00)$ & $14.50(13.93-15.00)$ & 0.232 \\
maximal dose (Gy) & $40.13(31.00-50.00)$ & $29.00(27.86-30.00)$ & 0.397 \\
tumor vol (cm $\left.{ }^{3}\right)$ & $10.67(5.04-16.28)$ & $14.54(7.47-22.40)$ & \\
\hline
\end{tabular}

* Data presented as mean $(95 \% \mathrm{Cl})$ unless otherwise indicated.

GKS. This patient, a 75-year-old man, had undergone GKS for an $18-\mathrm{cm}^{3}$ chordoma located primarily in the clivus (Fig. 5). In his case the lesion was prescribed 35 Gy to the $50 \%$ isodose line. Twenty months after GKS, he experienced persistent diplopia and gait disturbance as a result of radiation necrosis. The symptoms resolved completely after a 2-week course of corticosteroids, and at the latest follow-up examination, he was asymptomatic with stable tumor size.

\section{Illustrative Cases}

Case 1. A 70-year-old man was examined for persistent headache. A petroclival tumor was noted and partially removed by craniotomy. The pathological diagnosis was chordoma. The tumor margin received a GKS dose of $14 \mathrm{~Gy}$, but most of the tumor volume received $16 \mathrm{~Gy}$; the medial portion of the tumor received a lower dose to

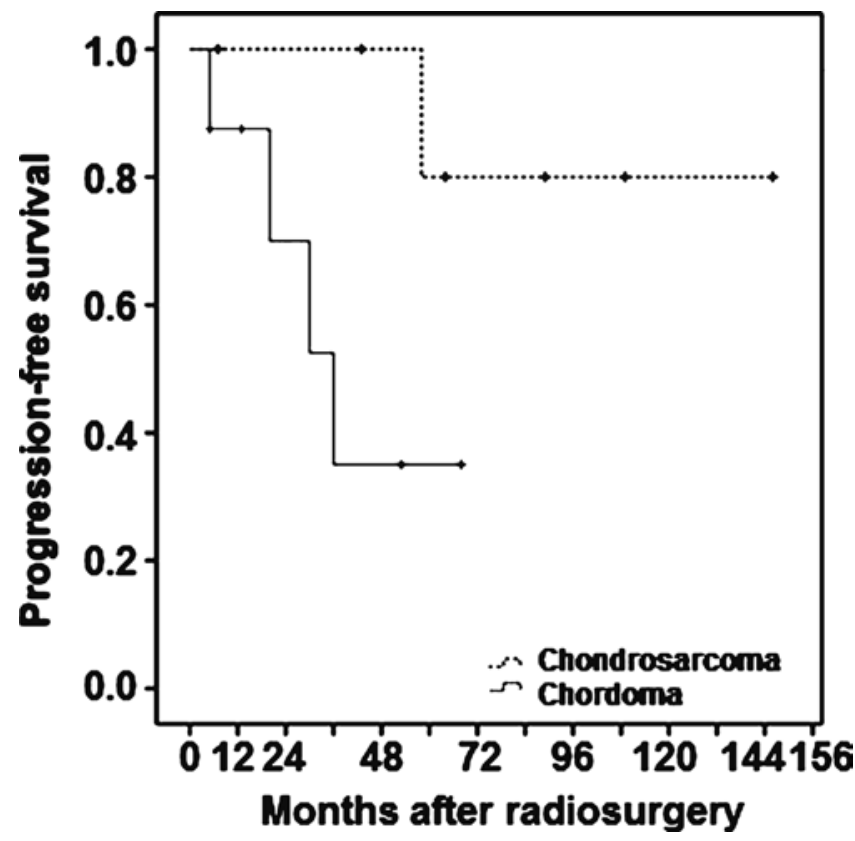

FIG. 1. Kaplan-Meier curves for progression-free survival among patients with chordoma $(n=5)$ and chondrosarcoma $(n=5)$. Rates were significantly better among patients with chondrosarcoma than among patients with chordoma $(p=0.04$, log-rank test). spare the brainstem. The tumor enlarged 20 months after GKS; enlargement was especially observed in the medial part of the tumor, where the dose was reduced (Tables 1 and 2; Fig. 6).

Case 6. A 48-year-old woman was examined for right third cranial nerve palsy. A middle fossa tumor was noted and subtotally removed by craniotomy. The specimen was pathologically diagnosed as chondrosarcoma. Postoperatively, she underwent GKS. The whole volume of the residual tumor was covered by a dose of 14 Gy to the margin of the tumor. The tumor had been controlled for 146 months as of the latest follow-up imaging (Tables 1 and 2; Fig. 7).

\section{Discussion}

Chordomas and chondrosarcomas are slow-growing,

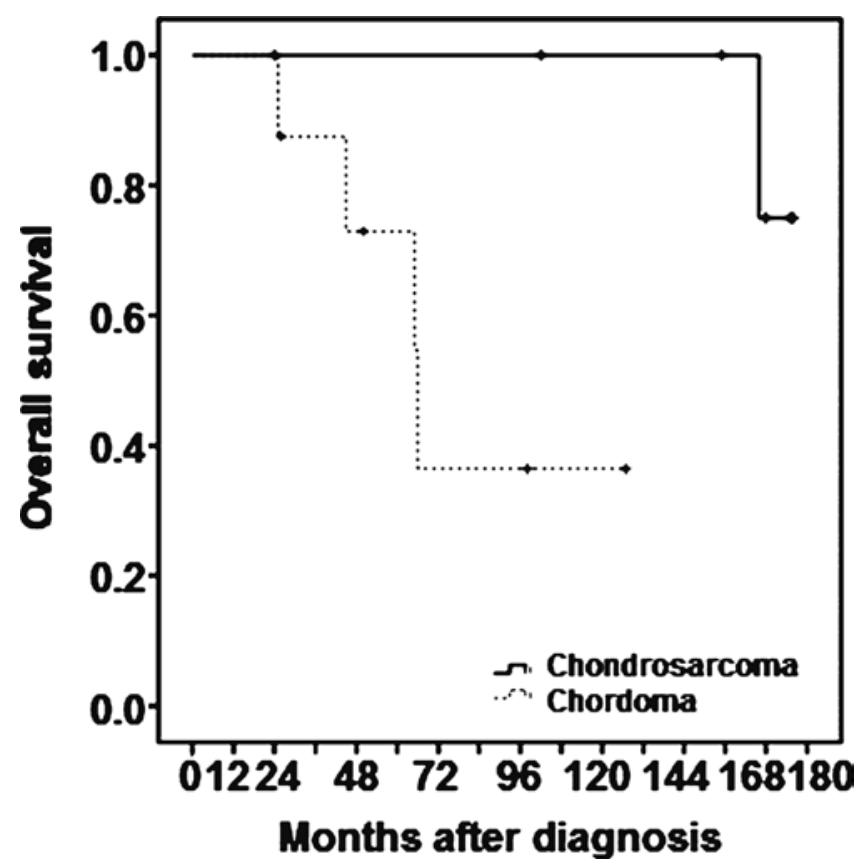

FIG. 2. Kaplan-Meier curves for overall survival among patients with chordoma $(n=5)$ and chondrosarcoma $(n=5)$. The 5 -year overall survival rates among patients with chordoma and chondrosarcoma were $72.9 \%$ and $100 \%$, respectively ( $p=0.03$, log-rank test). 


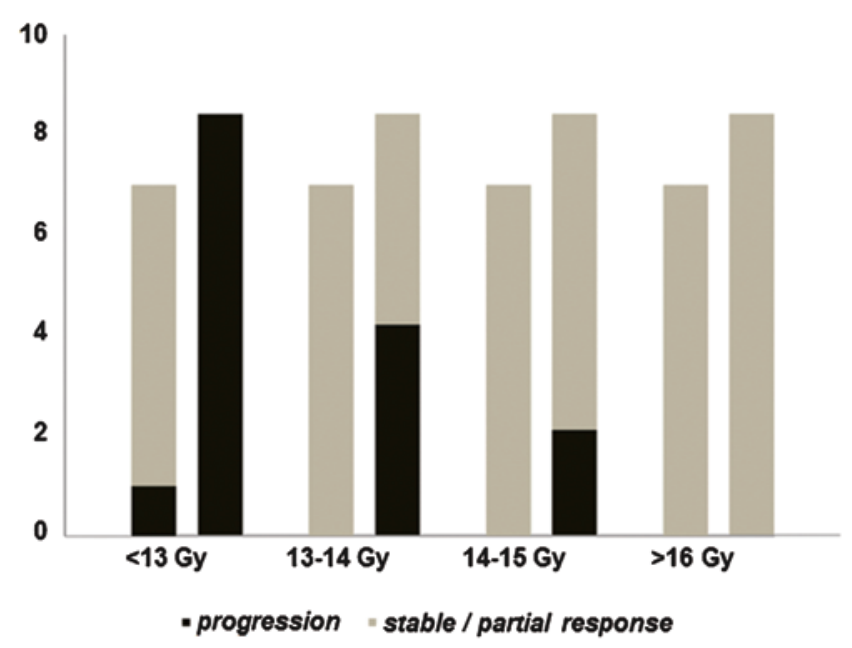

Fig. 3. Comparison of tumor progression, according to margin dose, between patients with chordoma (right bars) and chondrosarcoma (left bars).

locally invasive tumors. Chordomas arise from embryonic remnants of the notochord at the 2 extreme ends of the vertebral axis and comprise approximately $0.1 \%-0.2 \%$ of all primary intracranial neoplasms. ${ }^{2,17,25,30}$ Chondrosarcomas originate from primitive mesenchymal stem cells within the cartilaginous matrix of the skull base and represent only $0.02 \%$ of all intracranial neoplasms. ${ }^{2,17,25,30}$ The clinical presentations and radiological features of chordomas and chondrosarcomas are reported to be similar, but their clinical behavior, response to treatment, and outcomes differ. ${ }^{2,25,30}$ Chordomas are most commonly diagnosed during the patient's 2nd or 3rd decade of life, ${ }^{17}$ and $25 \%-40 \%$ occur in the sphenooccipital or skull base region, most commonly the clivus. ${ }^{17}$ Chondrosarcomas grow slowly and rarely metastasize ${ }^{11,17}$ and, similar to chordomas, are most commonly found in the sphenoid bone or clivus, at the base of the skull. However, the natural history of untreated clival chordomas is dismal, whereas chondrosarcomas usually do not metastasize until a very late stage..$^{8,11,17}$

The therapeutic options for these tumors include resection, fractionated radiotherapy, GKS, and proton beam radiotherapy ${ }^{10,13,14}$ Initial management is most commonly resection of these tumors; ;, $3,13,16,24,31$ however, because of their proximity to, or involvement with, critical structures, such as the critical vessels, cranial nerves, and brainstem, complete resection without significant complications is

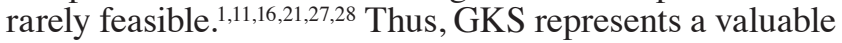
management option for patients with recurrent or residual chordoma or chondrosarcoma whose lesions failed to respond to initial resection and adjuvant radiation therapy. In the past, intracranial chordomas and chondrosarcomas have erroneously been grouped together and managed in the same way because of their many similarities. Moreover, because of the rarity of these malignancies, most clinical series published on the results of radiosurgery for these tumors have included a combination of chordoma and chondrosarcoma cases, despite their different histological origins. However, the results of the current series demonstrate a clear distinction in the radiosurgical outcomes and biological behavior of chordomas and chondrosarcomas.

\section{Rates of Progression-Free and Overall Survival After GKS}

Several reports of the comparative outcomes after GKS for these tumors have been published. Krishnan et al. reported that among 25 patients with chordoma and 4 patients with chondrosarcoma who underwent GKS, tumor progression was occurred in 7 (28\%) patients with chordoma, whereas tumor control was achieved in all $4(100 \%)$ patients with chondrosarcoma. ${ }^{20}$ Martin et al. reported on their radiosurgical experience in treating 18 patients with chordoma and 10 patients with chondrosarcoma. ${ }^{22}$ They noted that the actuarial 5-year rates $( \pm \mathrm{SD})$ of local tumor control for chordomas and chondrosarco-

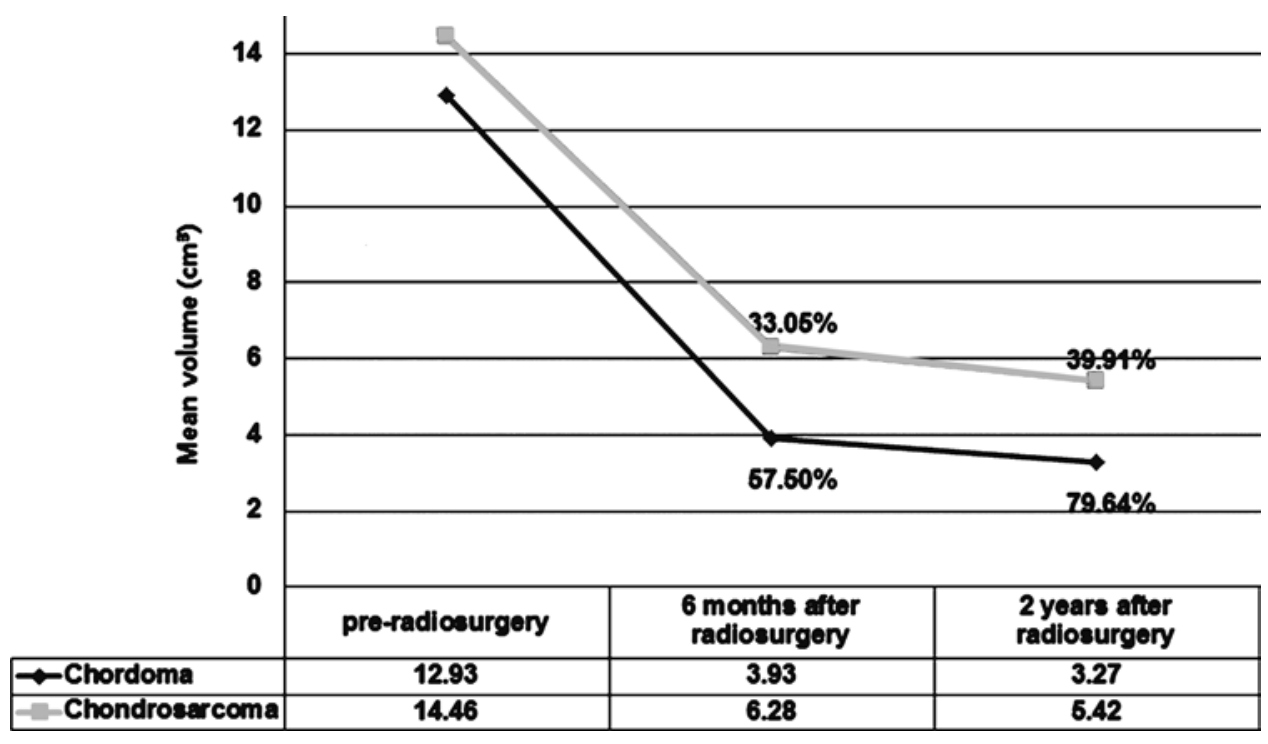

Fig. 4. Mean rates of tumor volume change. After 6 months, rates were $57.50 \%$ for chordoma and $33.05 \%$ for chondrosarcoma $(p=0.46)$; after 2 years, rates were $79.64 \%$ and $39.91 \%$, respectively $(p=0.05)$. 

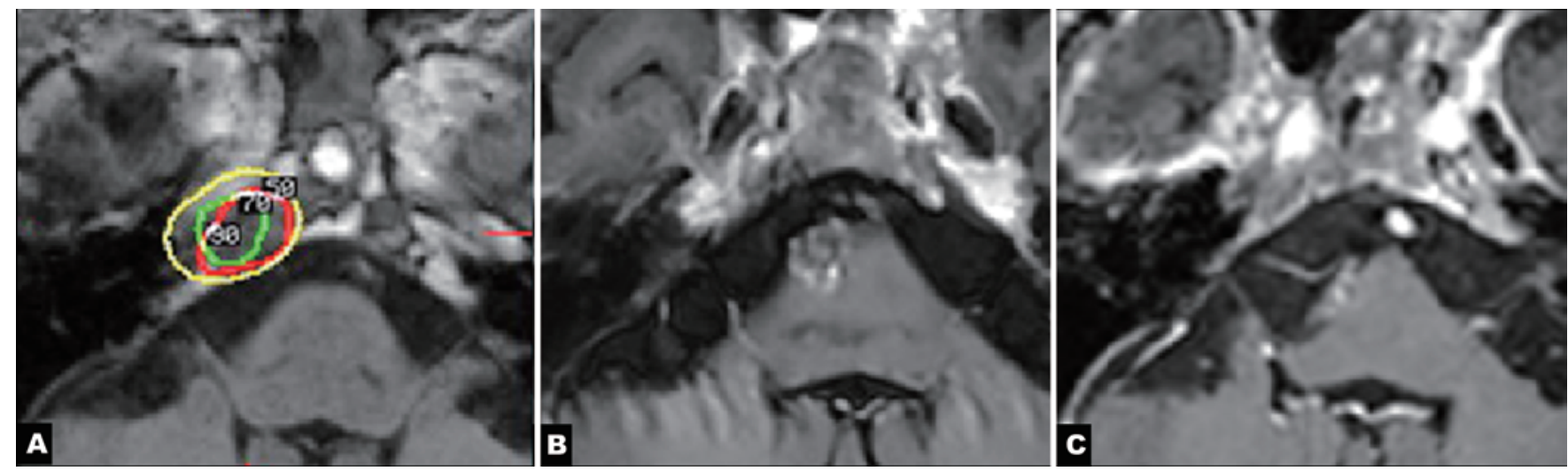

FIG. 5. A: Dose planning of radiosurgery for chordoma. Yellow indicates $50 \%$ isodose line; red indicates $70 \%$ isodose line; and green indicates $90 \%$ isodose line. B: Representative contrast-enhanced T1-weighted MR image 20 months after radiosurgery, showing an enhancing lesion extending into the brainstem. C: Contrast-enhanced T1-weighted MR image taken 53 months after radiosurgery, revealing absence of the lesion.

mas were $62.9 \% \pm 10.4 \%$ and $80 \% \pm 10.1 \%$, respectively. Moreover, Hasegawa et al. reported on 37 patients who underwent GKS:12 30 with chordoma and 7 with chondrosarcoma. They showed that the actuarial 5- and 10- year progression-free survival rates among patients with histologically confirmed chordoma were $42 \%$ and $32 \%$, respectively, whereas the 5-year progression-free survival rate among patients with chondrosarcoma was $80 \%$.
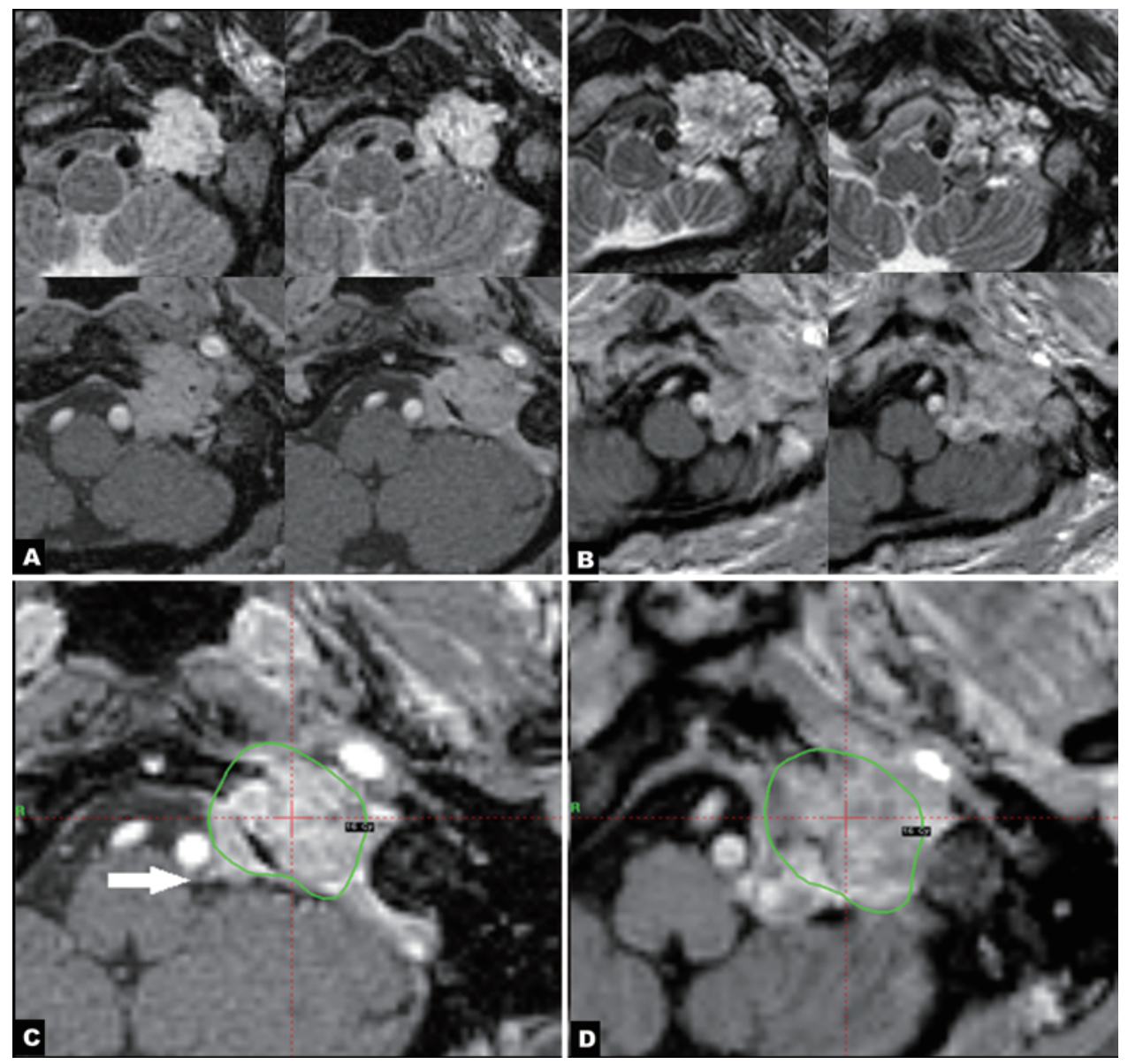

FIG. 6. Case 1: chordoma. Axial T2-weighted (A) and contrast-enhanced T1-weighted (C) MR images, showing a mass arising from the petroclival region extending into the brainstem before radiosurgery. Axial T2-weighted (B) and contrast-enhanced T1weighted (D) MR images showing progression of the treated lesion 20 months after radiosurgery. C: Most of the volume was covered by the margin dose of $16 \mathrm{~Gy}$; the delivered dose to the medial portion of the tumor was reduced to spare the brainstem (arrow). D: Gd-enhanced T1-weighted MR image obtained 20 months after radiosurgery, showing that the tumor continues to grow, especially where the radiation dose was reduced. 
J. H. Kim et al.
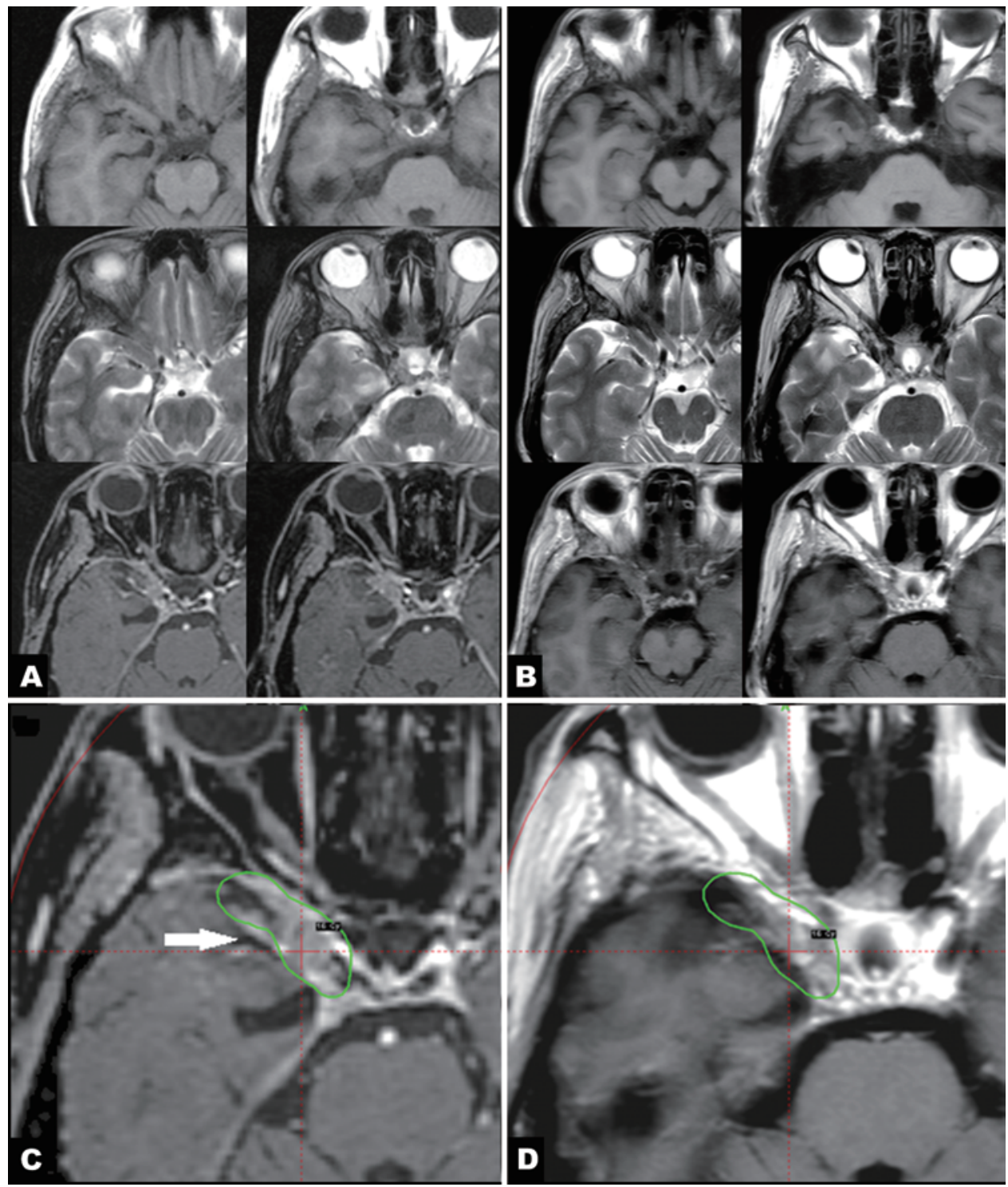

FIG. 7. Case 6: chondrosarcoma. A: Axial T1-weighted (upper), T2-weighted (center), and contrast-enhanced T1-weighted (lower) MR images, revealing a tumor around the cavernous sinus for which GKS was performed. B: Axial T1-weighted (upper), T2-weighted (center), and contrast-enhanced T1-weighted (lower) MR images, showing significant regression of the treated lesion 146 months after radiosurgery. C: The whole volume was well covered by a margin dose of 14 Gy but was not covered by a margin dose of $16 \mathrm{~Gy}$ because of its lateral portion (arrow). D: T1-weighted MR image with gadolinium enhancement obtained 146 months after radiosurgery, showing regression of the tumor.

Hence, local tumor control rate and prognosis seem to be generally better for patients with chondrosarcoma than for those with chordoma, and these results are similar to our findings. In our study, the 5-year progression-free survival rates after GKS for chordoma and chondrosarcoma were $35 \%$ and $80 \%$, respectively. Furthermore, our results revealed significantly better 5 -year progression-free survival rates among patients with chondrosarcoma. Combining these findings with those of the aforementioned studies, it seems that the clinical outcome after GKS for chordoma is poorer than that after GKS for chondrosarcoma; this finding is similar to the overall clinical outcomes for these tumors.

\section{Volume Changes After GKS}

GKS is traditionally evaluated by measuring patient survival times and local tumor control. To our knowledge, changes over time in tumor volume after GKS have not been previously reported for patients with chordoma and chondrosarcoma. Our study showed that 2 years after GKS, chordoma volume decreased significantly more than chondrosarcoma volume, indicating significant differences in the radiosurgical responses of these lesions.

Different tumors respond differently to radiosurgery, ${ }^{26}$ and differences in the volume change after GKS can be attributed to the tumor histology. ${ }^{6,18}$ Considering that chordomas are associated with substantially worse 
clinical outcomes than chondrosarcomas, it is possible that chordomas also have more aggressive malignant radiosurgical responses than chondrosarcoma in terms of the changes in tumor volume. The results of this study suggest that volume changes after GKS seem to be one of the differences between these 2 tumors.

\section{Optimal Radiation Dose}

When determining the appropriate radiation dose, both tumor control and prevention of radiation-induced complications are usually considered. However, for cases of chordoma and chondrosarcoma, little is known about the optimal radiation dose. Local failures constitute the main cause for treatment failure of intracranial chordoma and chondrosarcoma, ,,23 and several studies have demonstrated the value of achieving local control. One report regarding the radiosurgical results for 25 patients with chordoma and 4 patients with chondrosarcoma suggested that there were 2 types of treatment failure: in-field failure, in which the failure occurred within the prescription isodose volume, and out-of-field failure, in which the failure occurred outside the prescription isodose volume. ${ }^{20}$ The authors moreover analyzed the association between the patterns of failure and tumor doses and found that there were no in-field failures when the tumor margin dose was greater than $15 \mathrm{~Gy}$. Iyer et al. described a series of 22 patients who underwent stereotactic radiosurgery for cranial base chondrosarcoma. ${ }^{15}$ Of these patients, 2 experienced progression adjacent to the prescribed tumor volume and 1 experienced distant tumor progression. Together, the results of these previous studies all emphasize the effects of in-field failure, which is presumably associated with an inadequate radiation dose.

For controlling tumor growth within the planned treatment volume, it seems that a higher margin dose is crucial. Koga et al. reported on 16 lesions in 14 patients treated for skull base chordoma and chondrosarcoma; treatments with margin doses higher and lower than 15 Gy were defined as high-dose and low-dose treatments, respectively. ${ }^{19}$ The 5 -year progression-free survival rates for patients who underwent GKS with high and low margin doses were $80 \%$ and $14 \%$, respectively $(\mathrm{p}=0.005)$. Another report by Hasegawa et al. studied the factors associated with local tumor control among 46 lesions in patients with skull base chordomas or chondrosarcomas. ${ }^{12}$ In-field tumor progression developed in 10 (22\%) lesions in 9 patients, and the authors concluded that dose planning with a generous treatment volume to avoid failure at the tumor margin should be made at a margin dose of at least 15 Gy to achieve long-term control. Similarly, most authors have recommended that a margin dose of at least 15 Gy should be used to achieve local control of these types of tumors, without differentiating between tumors according to their biological behavior.

As mentioned earlier, because radiosurgical responses of chordoma and chondrosarcoma differ, doses should differ. However, the currently recommended radiation dose for these tumors is 15 Gy regardless of tumor type. On the basis of the individual patient data in our study, we found no tumor progression when margin doses greater than 16 Gy for chordoma and 14 Gy for chondrosarcoma were used. Thus, we believe that good local control can be achieved with the use of relatively low radiation doses for treatment of chondrosarcoma. Although the number of patients in our study was relatively low, on the basis of our results we conclude that radiosurgery, with a margin dose of more than $16 \mathrm{~Gy}$ for chordoma and more than 14 Gy for chondrosarcoma, might result in enhanced local tumor control with relatively few complications.

We have suggested a process for determining the optimal radiation dose for patients with intracranial chordoma or chondrosarcoma (Fig. 8). Most tumors require

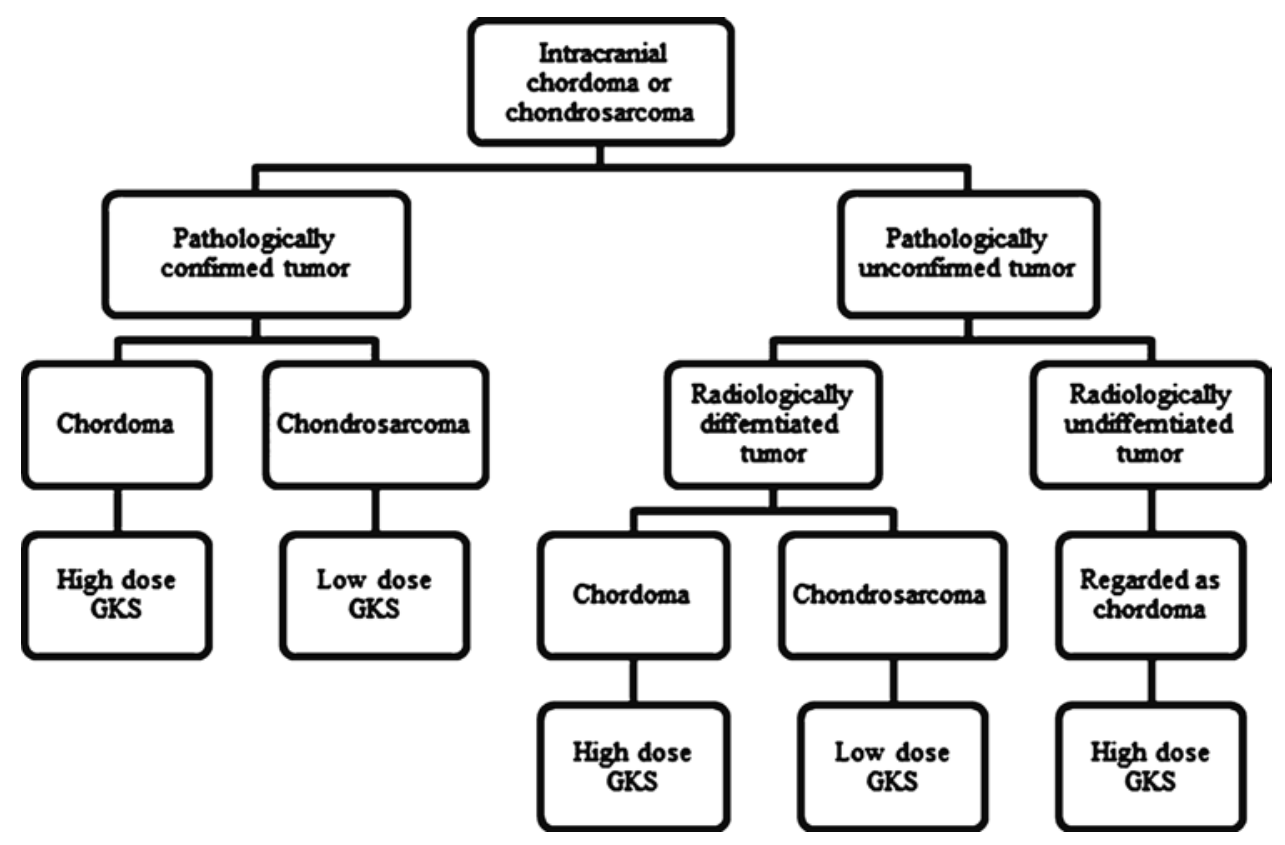

FIG. 8. Flow diagram of our proposed strategy for optimal radiation dose planning for patients with intracranial chordoma or chondrosarcoma. High-dose GKS: margin dose of 16 Gy to the tumor; low-dose GKS: margin dose of 14 Gy to the tumor. 
resection and pathological confirmation. However, the patient's condition might not allow for surgical treatment, such as craniotomy or biopsy under general anesthesia. If surgical treatment is not feasible, we try to distinguish these tumors radiologically. If radiological differentiation is also not possible, we recommend regarding the tumor as a chordoma and treating it accordingly to achieve better tumor control. We also believe that adjusting the GKS radiation dose for each individual tumor will help achieve better outcomes with a relatively low rate of radiationassociated complications.

\section{Conclusions}

Although surgical resection is currently the gold standard for treating intracranial chordomas and chondrosarcomas, GKS also plays a role in the treatment of these malignancies, especially for remnant or recurrent tumors. In this study, GKS showed acceptable rates of tumor control with relatively low rates of complications. However, the radiosurgical response differed among the tumors according to the biological behavior of each tumor. A higher margin dose was required to achieve control of chordomas, whereas relatively low doses were sufficient to achieve both tumor control and cranial nerve preservation for chondrosarcomas. The small number of cases in this study limits its interpretation; further followup studies and accumulation of cases are hence required to determine the optimal dose of GKS for patients with intracranial chordoma or chondrosarcoma.

\section{Acknowledgments}

We thank Eun Mi Bang, Ji Hyun An, and Gi Hong Kim from Yonsei Gamma Knife Center for assistance with this study.

\section{Disclosure}

The authors have no personal financial or institutional interest in any of the drugs, materials, or devices described in this study. This study was supported by a new faculty research seed money grant of Yonsei University College of Medicine for 2014 (2014-32-0027).

Author contributions to the study and manuscript preparation include the following. Conception and design: WS Chang. Acquisition of data: all authors. Analysis and interpretation of data: all authors. Drafting the article: Kim. Critically revising the article: all authors. Reviewed submitted version of manuscript: all authors. Approved the final version of the manuscript on behalf of all authors: WS Chang. Statistical analysis: all authors. Administrative/techni$\mathrm{cal} /$ material support: all authors. Study supervision: WS Chang.

\section{References}

1. al-Mefty O, Borba LA: Skull base chordomas: a management challenge. J Neurosurg 86:182-189, 1997

2. Almefty K, Pravdenkova S, Colli BO, Al-Mefty O, Gokden M: Chordoma and chondrosarcoma: similar, but quite different, skull base tumors. Cancer 110:2457-2467, 2007

3. Ammirati M, Bernardo A: Management of skull base chordoma. Crit Rev Neurosurg 9:63-69, 1999

4. Cho YH, Kim JH, Khang SK, Lee JK, Kim CJ: Chordomas and chondrosarcomas of the skull base: comparative analysis of clinical results in 30 patients. Neurosurg Rev 31:35-43, 2008

5. Colli BO, Al-Mefty O: Chordomas of the skull base: follow- up review and prognostic factors. Neurosurg Focus 10(3):E1, 2001

6. Debus J, Schulz-Ertner D, Schad L, Essig M, Rhein B, Thillmann CO, et al: Stereotactic fractionated radiotherapy for chordomas and chondrosarcomas of the skull base. Int J Radiat Oncol Biol Phys 47:591-596, 2000

7. Di Maio S, Temkin N, Ramanathan D, Sekhar LN: Current comprehensive management of cranial base chordomas: 10year meta-analysis of observational studies. Clinical article. J Neurosurg 115:1094-1105, 2011

8. Eriksson B, Gunterberg B, Kindblom LG: Chordoma. A clinicopathologic and prognostic study of a Swedish national series. Acta Orthop Scand 52:49-58, 1981

9. Fagundes MA, Hug EB, Liebsch NJ, Daly W, Efird J, Munzenrider JE: Radiation therapy for chordomas of the base of skull and cervical spine: patterns of failure and outcome after relapse. Int J Radiat Oncol Biol Phys 33:579-584, 1995

10. Feigl GC, Bundschuh O, Gharabaghi A, Safavi-Abassi S, El Shawarby A, Samii M, et al: Evaluation of a new concept for the management of skull base chordomas and chondrosarcomas. J Neurosurg 102 Suppl:165-170, 2005

11. Gay E, Sekhar LN, Rubinstein E, Wright DC, Sen C, Janecka IP, et al: Chordomas and chondrosarcomas of the cranial base: results and follow-up of 60 patients. Neurosurgery 36:887897,1995

12. Hasegawa T, Ishii D, Kida Y, Yoshimoto M, Koike J, Iizuka H: Gamma Knife surgery for skull base chordomas and chondrosarcomas. J Neurosurg 107:752-757, 2007

13. Hauptman JS, Barkhoudarian G, Safaee M, Gorgulho A, Tenn $\mathrm{S}$, Agazaryan N, et al: Challenges in linear accelerator radiotherapy for chordomas and chondrosarcomas of the skull base: focus on complications. Int J Radiat Oncol Biol Phys 83:542-551, 2012

14. Hug EB, Loredo LN, Slater JD, DeVries A, Grove RI, Schaefer RA, et al: Proton radiation therapy for chordomas and chondrosarcomas of the skull base. J Neurosurg 91:432-439, 1999

15. Iyer A, Kano H, Kondziolka D, Liu X, Niranjan A, Flickinger JC, et al: Stereotactic radiosurgery for intracranial chondrosarcoma. J Neurooncol 108:535-542, 2012

16. Kano H, Iqbal FO, Sheehan J, Mathieu D, Seymour ZA, Niranjan A, et al: Stereotactic radiosurgery for chordoma: a report from the North American Gamma Knife Consortium. Neurosurgery 68:379-389, 2011

17. Kano H, Lunsford LD: Stereotactic radiosurgery of intracranial chordomas, chondrosarcomas, and glomus tumors. Neurosurg Clin N Am 24:553-560, 2013

18. Kim JH, Jung HW, Kim YS, Kim CJ, Hwang SK, Paek SH, et al: Meningeal hemangiopericytomas: long-term outcome and biological behavior. Surg Neurol 59:47-54, 2003

19. Koga T, Shin M, Saito N: Treatment with high marginal dose is mandatory to achieve long-term control of skull base chordomas and chondrosarcomas by means of stereotactic radiosurgery. J Neurooncol 98:233-238, 2010

20. Krishnan S, Foote RL, Brown PD, Pollock BE, Link MJ, Garces YI: Radiosurgery for cranial base chordomas and chondrosarcomas. Neurosurgery 56:777-784, 2005

21. Maira G, Pallini R, Anile C, Fernandez E, Salvinelli F, La Rocca LM, et al: Surgical treatment of clival chordomas: the transsphenoidal approach revisited. J Neurosurg 85:784-792, 1996

22. Martin JJ, Niranjan A, Kondziolka D, Flickinger JC, Lozanne KA, Lunsford LD: Radiosurgery for chordomas and chondrosarcomas of the skull base. J Neurosurg 107:758-764, 2007

23. Muthukumar N, Kondziolka D, Lunsford LD, Flickinger JC: Stereotactic radiosurgery for chordoma and chondrosarcoma: further experiences. Int J Radiat Oncol Biol Phys 41:387392, 1998

24. Pallini R, Maira G, Pierconti F, Falchetti ML, Alvino E, Cimino-Reale G, et al: Chordoma of the skull base: predictors of tumor recurrence. J Neurosurg 98:812-822, 2003 


\section{Radiosurgery for intracranial chordoma and chondrosarcoma}

25. Pamir MN, Ozduman K: Analysis of radiological features relative to histopathology in 42 skull-base chordomas and chondrosarcomas. Eur J Radiol 58:461-470, 2006

26. Pan DH, Guo WY, Chung WY, Shiau CY, Liu RS, Lee LS: Early effects of Gamma Knife surgery on malignant and benign intracranial tumors. Stereotact Funct Neurosurg 64 (Suppl 1):19-31, 1995

27. Sen CN, Sekhar LN, Schramm VL, Janecka IP: Chordoma and chondrosarcoma of the cranial base: an 8-year experience. Neurosurgery 25:931-941, 1989

28. Uttley D, Moore A, Archer DJ: Surgical management of midline skull-base tumors: a new approach. J Neurosurg 71 (5 Pt 1):705-710, 1989

29. van den Bent MJ, Vogelbaum MA, Wen PY, Macdonald DR, Chang SM: End point assessment in gliomas: novel treatments limit usefulness of classical Macdonald's Criteria. J Clin Oncol 27:2905-2908, 2009
30. Yeom KW, Lober RM, Mobley BC, Harsh G, Vogel H, Allagio $\mathrm{R}$, et al: Diffusion-weighted MRI: distinction of skull base chordoma from chondrosarcoma. AJNR Am J Neuroradiol 34:1056-1061, 2013

31. Yoneoka Y, Tsumanuma I, Fukuda M, Tamura T, Morii K, Tanaka R, et al: Cranial base chordoma-long term outcome and review of the literature. Acta Neurochir (Wien) 150: 773-778, 2008

Manuscript submitted June 1, 2014

Accepted July 23, 2014

Please include this information when citing this paper: DOI: 10.3171/2014.7.GKS 141213.

Address correspondence to: Won Seok Chang, M.D., Yonsei University College of Medicine, 50 Yonsei-ro, Seodaemungu, Seoul 120-752, Republic of Korea.email: changws0716@yuhs.ac. 